\title{
Bilateral Duplex collecting system in Horse shoe kidney: An unusual association
}

\author{
Pramod K. Sharma, R. Sharma, Mukesh K. Vijay, Amit Goel, Anup K. Kundu \\ Department of Urology, Institute of Post graduate Medical Education and Research, Kolkata (West \\ Bengal), India
}

A 17 years unmarried female presented to Gastroenterology Department of our hospital for nonspecific symptoms of epigastric pain. The Ultrasonographic examination of the patient revealed triple kidney with horseshoe anomaly. She was then referred to us for further evaluation. We further proceed with Intravenous Urography (IVU) which demonstrated a bilateral duplex system in a asymmetrical horseshoe kidney (L shaped fusion). Left side kidney was vertically placed while the right side kidney was horizontally placed. One of the moieties was on the right side of vertebral column while the other moiety was overlying the vertebra and isthmus was on the left side of vertebra (Figure-1). A contrast enhanced computed tomography (CECT) scan confirmed the diagnosis of bilateral duplex system in horseshoe kidney (Figure-2). As the patient had non-specific, unrelated symptoms, patient was give symptomatic treatment for the same and was advised regular follow-up. Duplicated collecting systems (also known as duplex collecting systems) can be defined as renal units containing two pyelocaliceal systems that are associated with a single ureter or with double ureters. The prevalence of Partial duplication is $0.6 \%$, while complete duplication of ureters occurs in $0.2 \%$ of live births (1). When a single ureteral bud bifurcates before the ampulla bifurcates, a duplex kidney with a bifid renal pelvis or bifid ureter results (2). Congenital anomalies of the kidneys include a group of so-called fusion anomalies, in which both kidneys are fused together in early embryonic life. Fusion anomalies of the kidneys can generally be placed into 2 categories: [1] horseshoe kidney and its variants and [2] crossed fused ectopia. Horseshoe kidney is probably the most common fusion anomaly. The term horseshoe kidney refers to the appearance of the fused kidney, which results from fusion at one pole. Horseshoe kidney is the most common renal fusion anomaly, the incidence being about 1

Figure 1 - Intravenous Urogram (IVU) showing bilateral duplex collecting system in horseshoe kidney in which left renal moieties are vertically placed and one of the right sided moiety lies over the vertebra (marked by arrow) while the other one lies to the right of the vertebra. All the calyces are facing medially.

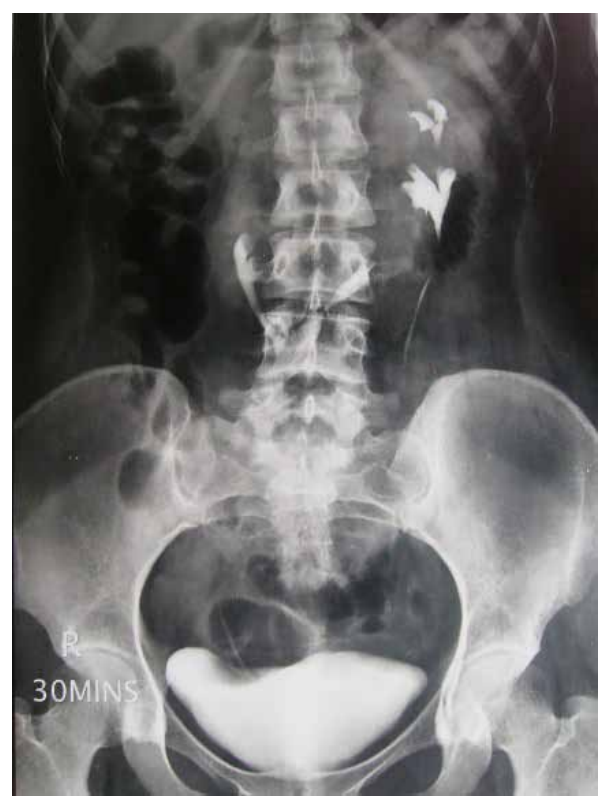


Figure 2 - Contrast Enhanced CT scan showing left side upper moiety ureter (marked by arrow), along with the left lower moiety and right side two moieties one placed right to vertebra and another lies over the vertebra.

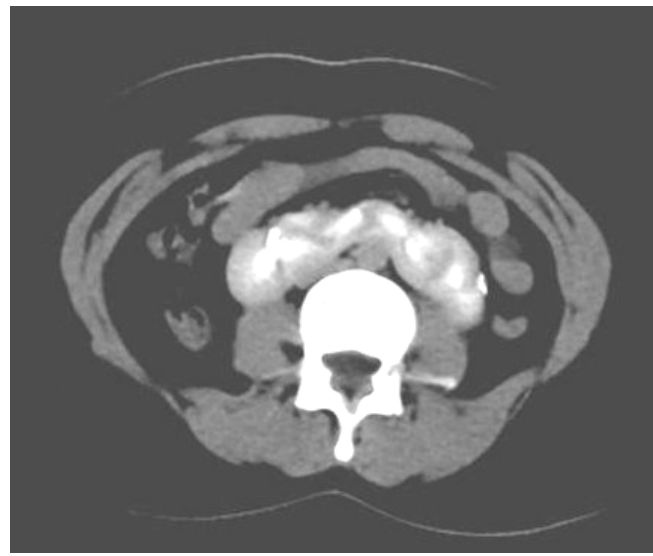

case per 400 persons worldwide. No clear evidence of a hereditary trait exists. In more than $90 \%$ of cases, fusion occurs along the lower pole. The renal pelvis are usually malrotated and lie anteriorly or laterally. Technically, the term horseshoe kidney is reserved for cases in which most of each kidney lies on one side of the spine. This is the symmetric horseshoe kidney (midline fusion). In asymmetric horseshoe kidney (L-shaped kidney), the fused part, or isthmus, lies slightly lateral to the midline (lateral fusion). Horseshoe kidney is generally differentiated from crossed fused ectopia, in which both fused kidneys lie on one side of the spine, and the ureter of the crossed kidney crosses the midline to enter the bladder. For the purpose of diagnosis, IVU is usually the first-line investigation, followed by CT scan or scintigraphy in cases with doubtful findings. The most common associated finding in horseshoe kidney is ureteropelvic junction (UPJ) obstruction, which occurs in up to 35\% of patients. It is the cause of most of the problems related to horse shoe kidneys. Obstruction is due to the high insertion of the ureter into the renal pelvis. The crossing of the ureter over the isthmus may also contribute to obstruction. Urinary stasis and stone disease also predispose the horseshoe kidney to infection, which occurs in 27 - 41\% of patients. Ascending infection from vesicoureteral reflux is another cause of infection in the horseshoe kidney. Only three cases with duplex pelvicalyceal system in horseshoe kidney are described in literature to the best of our knowledge (3-5).

\section{REFERENCES}

1. Glassberg KI, Braren V, Duckett JW: Suggested terminology for duplex systems, ectopic ureters and ureteroceles. J Urol. 1984; 132: 1153-4.

2. Bruno D, Delvecchio FC, Preminger GM: Successful management of lower-pole moiety ureteropelvic junction obstruction in a partially duplicated collecting system using minimally invasive retrograde endoscopic techniques. J Endourol. 2000; 14: 727-30.

3. Keskin S, Erdoğan N, Kurt A, Tan S, İpek A: Bilateral partial ureteral duplication with double collecting system in horseshoe kidney. Advances in Medical Sciences, 2009; 54: 302-4.

4. Christoffersen J, Iversen HG: Partial hydronephrosis in a patient with horseshoe kidney and bilateral duplication of the pelvis and ureter. Scand J Urol Nephrol. 1976; 10: 91-3.

5. Kuzel M, Makarewicz J, Musial S, Sarzynska M: Case of horseshoe kidney with bilateral double pelvis system and double ureters. Pediatr Pol. 1979; 54: 407-9.

Correspondence address:

Dr. Pramod Kumar Sharma

Room No. 508, Junior Doctor's Hostel, IPGME\&R and SSKM Hospital, 242, AJC Bose Road, Kolkata, West Bengal, 700020, India Email: surgpeekay@gmail.com

ARTICLE INFO

Int Braz J Urol. 2013; 39: 139-40

Submitted for publication: June 28, 2012

Accepted after revision: January 23, 2013 